\title{
Better Safe than Sorry: Interventional Radiology Should Be Prepared for the Coronavirus Disease 2019 Pandemic
}

\author{
1Department of Interventional Radiology, Christian Medical College, \\ Vellore, Tamil Nadu, India \\ 2Department of Diagnostic and Interventional Radiology, All India \\ Institute of Medical Sciences, Jodhpur, Rajasthan, India \\ ${ }^{3}$ Department of Interventional Neuroradiology, \\ Royal Preston Hospital, England, United Kingdom \\ J Clin Interv Radiol ISVIR 2020;4:38-41
}

Arul A.S. Babu ${ }^{1, \odot}$ Aswin Padmanabhan ${ }^{1}$ Pushpinder S. Khera ${ }^{2}$ Hemant Sonwalkar ${ }^{3}$

Address for correspondence Aswin Padmanabhan, MD, FVIR, Department of Interventional Radiology, Christian Medical College, Ida Scudder Road, Vellore 632004, Tamil Nadu, India (e-mail: aswinpaps@gmail.com).
Abstract
Keywords
- COVID-19
- interventional radiology
- PPE
- pandemic

Coronavirus disease 2019 (COVID-19) is a viral pneumonia that has plagued the world for much of the first quarter of 2020 and was recently declared a global pandemic. The caregivers must be aware of the disease and take steps to curb its transmission within the premises of our care settings (hospitals/clinics). In this article, we suggest a few proactive steps that can be taken at the institutional and departmental levels to break the transmission chain.

\section{Introduction}

Coronavirus disease 2019 (COVID-19) is a viral pneumonia caused by severe acute respiratory syndrome coronavirus 2 (SARS-CoV-2). This disease was discovered during an investigation of a cluster of pneumonia cases that did not respond to standard therapy in Wuhan City, China, in December $2019 .{ }^{1}$ Since then, the virus has rapidly spread across the world, in nearly 171 countries and territories around the world as per the latest reports of Johns Hopkins University, Baltimore, Maryland, United States. ${ }^{2}$ The World Health Organization (WHO) declared COVID-19 a global pandemic on March 11, 2020. ${ }^{3}$ This rapid spread is a testimony to the extremely infectious nature of the virus. The virus is capable of surviving in aerosols for hours and also on surfaces for days according to the latest study, adding to its virulence. ${ }^{4}$

Considering the novel nature of the virus, information regarding symptoms, mode of transmission, preventive measures, immunization strategies, and treatment options are evolving on a day-to-day basis. Interventional radiologists, by virtue of being involved in close proximity to patients, are at a greater risk of cross-infection than most other members of the radiology department. Hence, they must be aware as to how to protect themselves and prevent other staff members from getting infected while maintaining patient safety. In case of symptoms, timely and effective action should be taken.

\section{COVID-19}

Based on a few early reports emerging from South-East Asia, the average incubation period of the virus is 5 to 6 days, with $97.5 \%$ of the infected persons showing symptoms within 11.5 days of exposure. ${ }^{1} \mathrm{~A}$ WHO study of the initial outbreak in China suggests $80 \%$ of the cases had mild-to-moderate symptoms with a crude fatality rate (CFR) of 3.8\% among laboratory-confirmed COVID-19 cases. However, the disease is particularly severe among the elderly aged above 80 years (CFR: 21.9\%) and those with predicting medical conditions such as cardiovascular disease (CFR: 13.2\%), uncontrolled diabetes $(9.2 \%)$, respiratory disease $(8.0 \%)$, and hypertension (8.4\%). ${ }^{5}$ Similar data are also emerging from Italy, with a CFR of $9.6 \%$ for those aged between 70 and 79 years and $16.6 \%$ for those aged above 80 years. ${ }^{6}$ Another population group that is at a high risk is the health care workers (HCW), as they are the first-line defense of the community against the pandemic and potentially exposed to higher viral load. Approximately 3,000 HCWs were infected in China during the epidemic. ${ }^{7}$
Published online April 14, 2020
DOI https://doi.org/

$10.1055 / \mathrm{s}-0040-1710145$ ISSN 2457-0214.
(C)2020 by Indian Society of Vascular and Interventional Radiology
License terms

(두)(1) $\Theta \circledast$ 
Hence, it is clear that the elderly, those with preexisting conditions, and HCWs are at the highest risk of illness, and any planning should take this into consideration.

\section{Protection of Health Care Workers and Patients}

Policy regarding screening and management of individuals with suspicion of COVID-19 seems to be varying regionally and is evolving by the day. At this juncture, we would like to suggest the following steps to protect ourselves and our patients:

- Use of universal precautions and personal protective equipment (PPE) when dealing with suspected/ confirmed patients both during the procedure and during the consultation in out-patient clinics/ward. An HCW in an out-patient department is expected to use an N95 mask and gloves for personal protection so long as no aerosol-generating procedure (AGP) is performed. ${ }^{8}$ Goggles, though not part of recommendations, are a useful addition subject to availability.

- Screen and isolate patients who report symptoms similar to COVID-19 infection and refer them to a facility dealing with suspected COVID-19 patients.

- The department must have a policy in place regarding the imaging of suspected COVID-19 patients. A portable $\mathrm{X}$-ray machine stationed in the isolation ward is the best way to image such cases, as and when the need arises. CT (computed tomography) scan should only be used sparingly since it would require the patient to be shifted to the department, thereby potentially exposing staff to a risk of infection. This is particularly important in departments where interventional radiology (IR) laboratories and imaging equipment are located adjacent to each other.

- Delay or postpone nonemergency, elective procedures. Each hospital should develop its own guidelines on what constitutes elective procedures. The decision regarding the urgency of individual cases may be best taken through a multidisciplinary team meeting called on a virtual platform. The Society of Interventional Radiology (SIR) gives a sample classification of cases based on acuity that interventional radiologists can use as guidance. ${ }^{9}$

- Any HCW who has a history of travel to regions where local transmission of COVID-19 is reported should be encouraged to self-quarantine themselves for a period of 14 days even if asymptomatic and also be instructed to report to the COVID-19 facility if symptoms arise.

- Institutional infection control policy and recommendations of local governmental authorities, where applicable, should be strictly adhered to.

- HCWs are potential carriers of infection to people at their homes. Once back from work, special care needs to be taken to reduce contact with any articles or surfaces. It is advisable to use scrubs as much as possible in the hospital. If not, the regular clothes worn in the hospital may be put for washing immediately after return. A shower is advised before coming in contact with any family member. Materials carried to the hospital such as laptops and pens may be stowed separately in a safe place.
- Home quarantine for any suspicious symptoms is important. Unlike a few other pandemics which happened before, COVID-19 sheds infective virus a few days before the onset of symptoms. ${ }^{10}$ Thus, self-imposed quarantine if mildly symptomatic or after contact with a symptomatic patient (if not wearing proper PPE) is important.

\section{Protection of Staff and Personnel in the IR Suite}

Mossa-Basah et al ${ }^{11}$ have suggested some recommendations for the staff and personnel in the IR suite. These are as follows:

- Consider postponing elective procedures for a few days to weeks.

- Good surface cleaning of the equipment and room surfaces should be performed after every procedure using a chlorine-based disinfectant.

- Deep cleaning and fumigation of the room should be performed when a reverse transcriptase-polymerase chain reaction COVID-19 positive patient undergoes a procedure. In our institution, $1 \%$ solution of sodium hypochlorite is used for terminal cleaning of floors, walls, and high-touch surfaces, and 70\% isopropyl alcohol is used for equipment disinfection.

- Use disposable plastic covers over the detector and tube during imaging and intervention of all patients

- Screen all patients undergoing procedure for fever, respiratory symptoms, and travel history. All patients with respiratory symptoms should be made to wear a surgical mask prior to transfer to the IR suite.

- When possible, procedures on COVID-19 suspect/positive patients should be performed as the last procedure, and the IR suite should be thoroughly ventilated for at least 1 hour before the next procedure by using blowers or natural ventilation.

- Operators should be trained in donning and doffing of PPE. A dedicated area should be earmarked for donning of PPE before entering the IR suite.

- Special caution is to be exercised when performing an AGP such as thoracocentesis, pleural drain placement, or gastrostomy, or witnessing intubation/ extubation, airway suctioning, and so on. An extensive list of AGPs involving IRs is available on the SIR COVID-19 resources webpage. ${ }^{12}$ Appropriate PPE (disposable waterproof gowns, N95 or higher order respirator, eye protection such as goggles or visor mask) should be used when performing these procedures.

- Minimize the number of HCWs in the procedure room when performing a procedure on COVID-19 positive patients.

- Trainees' participation may need to be limited based on the availability of PPE.

- A patient's bed/stretcher should remain in the procedure room or the anteroom throughout the procedure. If not possible and if the bed/stretcher has to be kept in the hallway, label it as COVID-positive. The stretcher guard rails should be wiped down before transferring the patient.

- Reuse and resterilization of hardware used for COVID-19 suspect/confirmed patients should be avoided. When 
inevitable, these hardware should be double-autoclaved/ gas-sterilized as appropriate.

- On March 22, 2020, Indian Council for Medical Research (ICMR) has recommended hydroxychloroquine (HCQ) for chemoprophylaxis of COVID-19 among asymptomatic HCWs. A dosage of $400 \mathrm{mg}$ twice a day on day 1 followed by $400 \mathrm{mg}$ once weekly for the next 7 weeks is recommended. ${ }^{13}$ At the moment, large randomized control trials supporting the use of HCQ prophylaxis is lacking. In this regard, it is suggested that individual caution should be exercised and suggestions of a physician with experience in using HCQ be sought.

\section{IR Service at the Peak of the Epidemic}

As we approach the peak of the epidemic, hospitals are expected to be overrun with COVID-19 infected patients occupying most of the beds in the wards and the intensive care units (ICUs). Deaths are expected directly due to the COVID-19 infection. Deaths may also occur indirectly due to a lack of facilities and staff for preventable acute life-threatening illnesses such as myocardial infarction, trauma, and stroke and in the long run due to a lack of timely intervention for illnesses such as chronic kidney disease and malignancies.

All nonlife-threatening IR procedures will need to be postponed. All patient consultations should be performed on telephone or through video links to reduce contact. All multidisciplinary conferences should be conducted using remote teleconferencing tools. Emergency IR procedures such as stroke interventions and embolization for acute bleeders (including ruptured intracranial aneurysm) should be continued as long as possible. Hyperacute services such as trauma, cardiac, and neurovascular units may have to move to designated non-COVID hospitals. Changes to the working pattern of IR teams should be considered so that IR services continue to be available when IR team members fall sick or are required to self-isolate. Postprocedure protocols should be revised to limit the use of ICUs following IR procedures. High-risk procedures that require ICU admission in elderly patients should be discussed and, if possible, postponed to prioritize the resources for COVID-19 positive patients.

It is likely that physicians and HCWs would be redeployed to serve the patients affected with COVID-19. IR teams can be vital in supporting COVID-19 patients in the ICUs by inserting central venous catheters for fluid management, arterial catheters for blood gases, and drainage of pleural collections. In addition, given that interventional radiologists are good at managing patients, they may be asked to staff ICUs and be part of the frontline medical teams taking care of these patients.

\section{Support Each Other}

The deluge of patients and bad news on social media can have a severe impact on the morale of the staff. This has the potential to adversely affect the working environment. Kindness toward colleagues, understanding each team member's personal situation, nonjudgmental attitude, and a positive approach would help the team to get over the crisis.

\section{Conclusion}

The COVID-19 pandemic is a reality, and interventional radiologists as clinicians are at risk of coming in close contact with diagnosed and undiagnosed cases of COVID-19. The steps mentioned previously have been put into practice at our institutions to break the chain of transmission. Having said that, policies and guidelines are changing on a daily basis and we need to be constantly updated with the information. We would also like to direct the attention of the reader to recent COVID-19 resources published by the SIR. ${ }^{12}$ Though many of us may not work in setups actively involved in the management of COVID-19, as these cases are predominantly managed in government facilities across India, our centers may be the points of primary contact for symptomatic patients and it may be prudent to practice caution in this hour of a pandemic. As we take this pandemic head-on, we are "better safe than sorry."

\section{Conflict of Interest}

None declared.

\section{References}

1 Zhu N, Zhang D, Wang W, et al; China Novel Coronavirus Investigating and Research Team. A novel coronavirus from patients with pneumonia in China, 2019. N Engl J Med 2020;382(8):727-733

2 Johns Hopkins University. COVID-19 Data Center. Available at: https://coronavirus.jhu.edu/. Accessed March 24, 2020

3 World Health Organization. WHO Director-General's opening remarks at the media briefing on COVID-19. Available at: https://www.who.int/dg/speeches/detail/who-directorgeneral-s-opening-remarks-at-the-media-briefing-on-covid19---11-march-2020. Accessed March 17, 2020

4 New England Journal of Medicine. Aerosol and Surface Stability of SARS-CoV-2 as Compared with SARS-CoV-1. Available at: https://www.nejm.org/doi/full/10.1056/NEJMc2004973. Accessed March 18, 2020

5 World Health Organization. Report of the WHO-China Joint Mission on Coronavirus Disease 2019 (COVID-19). Available at: https://www.who.int/docs/default-source/coronaviruse/ who-china-joint-mission-on-covid-19-final-report.pdf

6 Therese B. One Italian ICU Stressed to Breaking Point by COVID-19. Available at: https://www.medscape.com/ viewarticle/926804. Accessed March 17, 2020

7 Adams JG, Walls RM. Supporting the health care workforce during the COVID-19 global epidemic. JAMA 2020 (e-pub ahead of print);doi:10.1001/jama.2020.3972

8 Novel Coronavirus Disease 2019 (COVID-19): Guidelines on rational use of Personal Protective Equipment. Available at: https://www.mohfw.gov.in/pdf/GuidelinesonrationaluseofPersonalProtectiveEquipment.pdf. Accessed March 30, 2020

9 Society of Interventional Radiology. COVID-19 case classification. Available at: https://www.sirweb.org/practice-resources/covid-19-toolkit/covid-19-case-classifcation. Accessed March 30, 2020 
10 Bai Y, Yao L, Wei T, et al. Presumed asymptomatic carrier transmission of COVID-19. JAMA 2020 (e-pub ahead of print); doi 10.1001/jama.2020.2565

11 Mossa-Basah M, Meltzer CM, Kim DC, Tuite JK, Kolli P, Tan BS. Radiology department preparedness for COVID-19: radiology scientific expert panel. Radiology 2020 (e-pub ahead of print). doi;/radiol.2020200988

12 Society of Interventional Radiology. COVID-19 resources for IR. Available at: https://www.sirweb.org/practice-resources/ covid-19-resources/. Accessed March 30, 2020
13 Indian Council for Medical Research. Recommendation for empiric use of hydroxy-chloroquine for prophylaxis of SARS-CoV-2 infection. Available at: https://icmr.nic.in/sites/default/files/ upload_documents/HCQ_Recommendation_22March_final_ MM_V2.pdf Accessed March 30, 2020 